\title{
REVISORES AÑO 2009
}

\section{INTERNACIONALES}

Adeyinka Tella - University of Botswana (BOT) - tellayinkaedu@yahoo.com Al Bellamy - Eastern Michigan University (USA) - a.bellamy@.umich.edu Alberto Sáez - DYSLE (SWI) - uni ch@yahoo.com

Alex Kozulin - Int. Cent. Enhancement of Learning Potential (ISR) - kozulin@post.tau.ac.il Ali Eissa Mourad - Zagazig University (EGY) - drwesam20002000@yahoo.com Allan B. I. Bernardo - De la Salle University (PHI) - bernardoa@dlsu.edu.ph Allen Thurston - The Stirling Institute of Education (UK) - allen.thurston@stir.ac.uk Amy Shapiro - Universtiy of Massachusetts (USA) - ashapiro@umassd.edu Ana Couceiro - Universidade de Coimbra (PT) - apcouceiro@fpce.uc.pt Analia Chiecher - Universidad Nacional de Río Cuarto (ARG) - achiecher@hum.unrc.edu.ar Anneke Vrugt - University of Amsterdam (NETH) - A.J.Vrugt@uva.nl Annemie Desoete - University of Gent (BEL) - anne.desoete@ugent.be Assimina Tsibidaki - University of the Aegean (GRE) - tsimpidaki@rhodes.aegean.gr Babatunde Adeyemi - Obafemi Awolowo University (NIG) - adeyemibabs2003@yahoo.com Bola Ogunyemi - Olabisi Onabanjo University (NIG) - jibolaogunyemi@yahoo.com Carlos R. González - Universidad Autónoma de Yucatán (MEX) - garriaga@tunku.uady.mx Carmen Cárdenas - magdagis43@hotmail.com

Christian Morasse - Collège de Montréal (CAN) - morasse.christian@gmail.com Christopher Was - Kent State University (USA) - cwas@kent.edu Cruz García - Universidad Autónoma Metropolitana (MEX) - csh96327267@titlani.uam.mx Daniel C. Moos - Gustavus Adolphus College (USA) - dmoos@gustavus.edu Daniel Heggs - University of Wales Institute (UK) - dheggs@uwic.ac.uk Debra Pepler - York University (CAN) - pepler@yorku.ca Eleftheria Gonida - Aristotle University of Thessaloniki (GRE) - gonida@psy.auth.gr Elena F. Ruiz - Instituto Politécnico Nacional (MEX) - elen fruiz@yahoo.com.mx Erika Cunningham - University of Dundee (UK) - eecunningham@dundee.ac.uk Fernando Hitt - Université du Québec (CAN) - hitt.fernando@uqam.ca Fernando Iriarte - Universidad del Norte (COL) - firiarte@uninorte.edu.co Gloria C. Henao - Universidad de San Buenaventura (COL) - gloriach@une.net.co Guadalupe Acle - Universidad Nacional Autónoma de México (MEX) - gaclet@servidor.unam.mx Héctor Ponce - Universidad Santiago de Chile (CHL) - hponce@usach.cl Heinke Roebken - Oldenburg University (GER) - h.roebken@uni-oldenburg.de Helen Cowie - University of Surrey-Roehampton (UK) - h.cowie@surrey.ac.uk Huy Phan - The University of New England (AUS) - hphan2@une.edu.au Irene D. Muria - Universidad Nacional Autónoma de México (MEX) - imuria@servidor.unam.mx Jared Bartels - University of Central Missouri (USA) - bartels@ucmo.edu Jason Osborne - North Carolina State University (USA) - jason osborne@ncsu.edu Jeny Mercer - University of Wales Institute (UK) - JMercer@uwic.ac.uk Joanna Kosmala-Anderson - Coventry University (UK) - j.kosmala-anderson@coventry.ac.uk Jose L. Villegas - Universidad de Los Andes (COL) - joselovi@yahoo.es Kemrai Bhatta - Tribhuvan University (NEP) - bhatta khem123@yahoo.com Kerim Gundogdu - Atatürk University (TUR) - gundogduk@gmail.com, kerim@atauni.du.tr Lalage Sanders - University of Wales Institute (UK) - Isanders@uwic.ac.uk Layla Tercanlioglu - University of Ataturl (TUR) - leyla@atauni.edu.tr 
Lucy Barnard - Texas Tech University - Im.barnard@ttu.edu

Luis Radford - Laurentian University (CAN) - Iradford@laurentian.ca

María A. Zavala - Universidad de Guanajuato (MEX) - alicia zavala2@yahoo.com.mx

María C. Ramírez - Universidad Autónoma de Yucatán (MEX) - rdorant@uady.mx

María Cardelle-Elawar - Arizona State University (USA) - mcardelle@asu.edu

Michael Michaelides - European University Cyprus (CYP) - michaelides@cycollege.ac.cy

Michaela Glaeser - University of Freiburg (GER) - michaela.glaeser-zikuda@ezw.uni-freiburg.de

Panagiotis Stamatis - University of the Aegean (GRE)- stamatis@rhodes.aegean.gr

Paul D. Bell - East Carolina University (USA) - bellp@ecu.edu

Paul Sander - University of Wales Institute (UK) - psander@uwic.ac.uk

Rod Nicolson - University of Sheffield (UK) - r.nicolson@sheffield.ac.uk

Ruben Edel - Universidad Veracruzana (MEX) - redel@uv.mx

Sally M. Reis - University of Connecticut (USA) - sally.reis@uconn.edu

Seth Parsons - George Mason University (USA) - sparson5@gmu.edu

Shaun Helman - University of Wales Institute (UK) - shelman@uwic.ac.uk

Sonia Fuentes - Universidad Central de Chile (CHI) - sfuentes@ucentral.cl

Sotiria Tzivinikou - Aristotle University of Thessaloniki (GRE) - tzivisot@nured.auth.gr

Teresa Castillo - Universidad de Chile (CHI) - tcastill@med.uchile.cl

Thomas McLaughlin - Gonzaga University (USA)- mclaughlin@gonzaga.edu

Thomas McLaughlin - Gonzaga University Spokane (USA) - mclaughlin@gonzaga.edu

Todd McElroy - Appalachian State University (USA) - mcelroygt@appstate.edu

Wondimu Ahmed - University of Groningen (NETH)- w.ahmed@rug.nl

Yiulian Liu - Southern Illinois University (USA) - yliu@siue.edu

Zsuzsanse Szabo - zsuzsanna25@gmail.com

\section{NACIONALES}

Alexander Maz - Universidad de Córdoba - ma1mamaa@uco.es

Ana García - Universidad de Granada - berben@ugr.es

Ana María de Caso - Universidad de León - amcasf@unileon.es

Ana Soldevila - Universitat de Lleida - soldevila@pip.udl.cat

Ángela Barrios - Universidad Autónoma de Madrid - angela.barrios@uam.es

Antonio Codina - Universidad de Almería - acodina@ual.es

Antonio Marín - Universidad de Granada - anmarin@ugr.es

Antonio Valle - Universidad A Coruña - vallar@udc.es

Asunción Bosch - Universidad de Almería - mabosch@ual.es

Bárbara Scandroglio - Universidad Autónoma de Madrid - barbara.scandroglio@uam.es

Belén López-Pérez - Universidad Autónoma de Madrid - bln Ipz@hotmail.com

Benito León - Universidad de Extremadura - bleon@unex.es

Carlos Monereo - Universidad Autónoma de Barcelona - carles.monereo@uab.es

Carmen Ferrándiz - Universidad de Murcia - carmenfg@um.es

Carmen Hernández - Universidad de la Laguna - cherjo@ull.es

César Coll - Universidad de Barcelona - ccoll@ub.edu

Clemente Franco - Universidad de Almería - cfranco@ual.es

Clemente Rodríguez - Universidad de Granada - clerosa@ugr.es

Concepción Iriarte - Universidad de Navarra - ciriarte@unav.es

Eduardo Fernández - Universidad de Granada - efharo@ugr.es

Elena Barberá - Universidad Oberta de Cataluña - ebarbera@campus.uoc.es 
Elena García-Baamonde - Universidad de Extemadura-mgarsan@unex.es

Encarnación Castro - Universidad de Granada - encastro@ugr.es

Enrique Castro - Universidad de Granada - ecastro@ugr.es

Esperanza Rocabert - Universidad de Valencia - esperanza.rocabert@uv.es

Fernando Justicia - Universidad de Granada - justicia@ugr.es

Francisca Serrano - Universidad de Granada - fdserran@ugr.es

Francisco Gil - Universidad de Almería -fgil@ual.es

Francisco J. Peralta - Universidad de Almería - franperas@terra.es

Francisco Rivas - Universidad de Valencia - francisco.rivas@uv.es

Francisco Ruiz - Universidad de Granada - fcoruiz@ugr.es

Fuensanta Cerezo - Universidad de Murcia - fcerezo@um.es

Gemma Filella - Universitat de Lleida-gfilella@pip.udl.es

Isabel Romero - Universidad de Almería - imromero@ual.es

Isidoro Segovia - Universidad de Granada - isegovia@ugr.es

Jesús Alonso - Universidad Autónoma de Madrid - jesus.alonso@uam.es

Jesús Gallardo - Universidad de Granada - gamu@arrakis.es

Jorge Jiménez - Universidad de Granada - jirodriguez@ugr.es

José M. Moreno - Universidad de Extremadura-jimmanso@unex.es

José Arias - universidad de Oviedo - arias@uniovi.es

José C. Nuñez - Universidad de Oviedo - jcarlosn@uniovi.es

José D. Villarroel - Universidad País Vasco - txomin.villarroel@ehu.es

José Gutiérrez - Universidad de Granada - iguti@ugr.es

José L. Arco - Universidad de Granada - jlarco@ugr.es

José L. González - Universidad de Málaga - gmari@uma.es

José M. Martínez - Universidad de Almería - jvicente@ual.es

José Ma Avilés - Universidad de Valladolid - imaviles@psi.uva.es

José Ma. Gil - Universitat Jaume Primero -jgil@psi.uji.es

José Madariaga - Universidad del País Vasco - josetxu.madariaga@ehu.es

Jose María Augusto - Universidad de Jaén - jaugusto@ujaen.es

José María Román - Universidad de Valladolid - jmroman@psi.uva.es

Juan A. Moriana - Universidad de Córdoba - ed1moeli@uco.es

Juan C. Pérez-González - UNED - jcperez@edu.uned.es

Juan M. Fernández - Universidad de Granada - fmillan@cop.es

Lucía Herrera - Universidad de Granada - luciaht@ugr.es

Ma Carmen Pichardo - Universidad de Granada - pichardo@ugr.es

Ma Consuelo Cañadas - Universidad de Granada - mconsu@ugr.es

Mạ Dolores López - Universidad de Granada - dlopezj@ugr.es

Mạ José González - Universidad de Cantabria - mariaj.gonzalez@unican.es

Mạ Luisa de la Morena - Universidad de Málaga - morena@uma.es

Mạ Mar García - Universidad de Almería - miguelangellabella@gmail.com

Mạ Trinidad Sánchez - Universidad de Castilla La Mancha - MTrinidad.Sanchez@uclm.es

Mà. J. González - Universidad de Málaga - valenzu@uma.es

Manuel Álvarez - Universidad de Barcelona - alvarez.m@ub.edu

Manuel Soriano - Universitat de Valencia - manuel.soriano@uv.es

María Cañadas - Universidad de Granada - mconsu@ugr.es

María Fernández - Universidad de Granada - mariafc@ugr.es

María Márquez - Universidad Autónoma de Madrid - maria.marquez@uam.es

Marta Molina - Universidad de Granada - martamg@ugr.es 
Melanie Shores - Universidad Autónoma de Barcelona-mshores@uab.edu

Nuria Aris Redó - Universitat Internacional de Catalunya - nuria@cir.uic.es

Pablo Fernández - Universidad de Málaga - berrocal@uma.es

Pablo Flores - Universidad de Granada - pflores@ugr.es

Pedro Gómez - Universidad de Granada - pgomez@valnet.es

Pilar Sánchez - Universidad de Almería - psanchez@ual.es

Rafael Camino - Hospital Universitario Reina sofía de Córdoba - rafacamino@gmail.com

Raquel Fidalgo - Universidad de León-rfidr@unileon.es

Raquel Palomera - Universidad de Cantabria - palomerr@unican.es

Rosario Cabello - Universidad de Málaga-rcabello@uma.es

Santiago Pelegrina - Universidad de Jaén - spelegri@ujaen.es

Santiago Ramirez - Universidad de Granada - sramirez@ugr.es

Sol Andrés - Universidad de Alcalá de Henares - soledad.andres@uah.es

Sonia Lara - Universidad de Navarra - slara@unav.es

Susana Lucas - Universidad de Valladolid - sulum@psi.uva.es

Tamara Polo - Universidad de Granada - tpolo@ugr.es 